\title{
3D Chemical mapping using terahertz pulsed imaging
}

\author{
Yao-Chun Shen, Philip F. Taday, David A. Newnham, Michael C. Kemp, and Michael Pepper \\ TeraView Limited, Platinum Building, St John's Innovation Park, \\ Cambridge CB4 0WS, United Kingdom
}

\begin{abstract}
We report the use of a terahertz pulsed imaging technique for three-dimensional chemical mapping. Terahertz radiation reflected from a sample was measured pixel-by-pixel in time domain using a terahertz pulsed imaging system developed at TeraView Ltd, UK. The recorded terahertz waveforms were then transformed into frequency domain using time-partitioned Fourier transform. Structural maps of samples were obtained by analyzing the terahertz time-domain data whilst chemical maps were obtained from terahertz spectral data sets. For a sample comprising chemical A at the surface of a polyethylene pellet and chemical B buried inside the pellet, we have separated the component spatial patterns of the two chemicals using their spectral fingerprints. The reconstructed three-dimensional chemical maps not only locate the chemicals in the object, but also identify each chemical. We also demonstrate the capabilities of terahertz pulsed imaging for non-destructive analysis of coating thickness and quality, and for detecting and identifying explosive materials such as RDX.
\end{abstract}

Keywords: chemical mapping, terahertz, spectroscopic imaging, three-dimensional, pharmaceutical, security screening

\section{INTRODUCTION}

Chemical mapping is an exciting new analytical advance that provides comprehensive information characterising complex heterogeneous samples. The basis of chemical mapping is the acquisition of a three-dimensional data set where two axes describe vertical and horizontal spatial dimensions, and the third axis represents the spectral frequency dimension. In the near- and mid-infrared frequency range, Fourier-transform infrared and Raman spectroscopy have been successfully developed for chemical mapping [1-3]. However, many materials such as pharmaceutical tablet coatings are opaque to infrared light. Therefore conventional infrared technique is mostly suitable for mapping out the surface distributions of chemicals, and thus is a two-dimensional chemical mapping technique. The chemical distribution in the depth direction cannot be accessed because infrared light cannot penetrate into the sample.

Terahertz pulsed imaging (TPI) is a newly-developed technique which utilizes the terahertz region of the electromagnetic spectrum $\left(10-330 \mathrm{~cm}^{-1}\right.$, or $\left.300 \mathrm{GHz}-10 \mathrm{THz}\right)$. In this frequency range many chemicals show characteristic spectral fingerprints and many packaging and coating materials are semi-transparent [4]. In addition, TPI directly measures the transient electric field, not simply the intensity of the terahertz radiation. This coherent detection scheme not only yields the terahertz signal with excellent signal-to-noise ratio and high dynamic range, but also allows both absorption coefficients and refractive indices to be obtained without the need to apply the Kramers-Kronig dispersion relationship [5-11].

In a TPI measurement, the terahertz waveform for each pixel was recorded as a function of optical time delay. This provides a three-dimensional (3D) data set where two axes describe vertical and horizontal spatial dimensions, and the third axis represents the time delay (depth) dimension. Applying a Fourier transform to the time-domain TPI data yields terahertz spectral data for each pixel. This gives in total a four-dimensional data set including vertical and horizontal spatial dimensions, the time (depth) dimension, and the spectral frequency dimension. The terahertz waveforms, which contain vital information about the refractive indices of the layered structures, have been previously used for nondestructive analysis of tablet coating thickness [12] and for the clinical imaging of basal-cell skin carcinoma ex vivo and in vivo [13]. Terahertz spectral data have also been analyzed for chemical mapping of heterogeneous mixtures and for detecting and identifying explosive [14]. In this paper, we show that combining analysis of both time-domain and frequency domain TPI data gives a true 3D chemical mapping of a sample. 
In the following sections we first briefly discuss the TPI technique (Section 2). In Section 3 we will concentrate on time-domain TPI data analysis, while in Section 4 we mainly discuss the spectral analysis. Example 3D chemical mapping images are presented in Section 4.

\section{MATERIALS AND METHOD}

High-density polyethylene (PE) powder was obtained from Chesham Chemicals, UK (particle size $<80 \mu \mathrm{m}$ ). Samples of $\alpha$-Lactose monohydrate and tartaric acid powders were purchased from Sigma-Aldrich, UK, and milled to fine powders (particle size $<100 \mu \mathrm{m}$ ) before use. Circular pellets were formed by compressing powder samples under 2tons using a hydraulic press (Specac, UK).

In all measurements, we used a TPI ${ }^{\mathrm{TM}}$ scan system (TeraView Ltd, UK) operating in rapid scan mode [12]. In this study, the terahertz radiation reflected from a sample was measured in time domain over an optical delay range of about $5 \mathrm{~mm}$, providing a spectral resolution of $1 \mathrm{~cm}^{-1}$ over the spectral range $5-80 \mathrm{~cm}^{-1}$. Terahertz images were obtained by raster scanning the terahertz beam at $100 \mu \mathrm{m}$ steps across the sample, which was mounted at the terahertz focus point. We show in the following sections that the time-domain terahertz images obtained contain all necessary information for mapping out the spatial distributions of the chemical components in a sample.

\section{TIME-DOMAIN TPI DATA FOR COATING ANALYSIS}

Coatings perform a wide variety of functions and pharmaceutical tablet coatings are actually now one of the preferred routes to control the release of active drug ingredients in the body. If the coating is non-uniform or has defects then the desired dose delivery and bioavailability can be compromised. Previous work [12] has demonstrated that TPI is a powerful tool for non-destructive determination of tablet coating thickness. Here we report further experimental results demonstrating that TPI is also a powerful tool for non-destructive inspection of subsurface structures and for assaying the interface quality of layered structures.

\subsection{Non-destructive detection of buried structures}

Figure 1(a) shows an example time-domain terahertz image recorded with $\mathrm{TPI}^{\mathrm{TM}}$ scan. The sample is a PE pellet containing a buried piece of weighing paper (Schleicher \& Schuell ${ }^{\circledR}, 40-50 \mu \mathrm{m}$ thick). The weighing paper is located 1.8 $\mathrm{mm}$ beneath the PE pellet surface and is therefore not visible. However, the paper is clearly seen in both the B-scan terahertz image and the waveform signal shown in Figures 1(c) and (d) respectively. This is because the terahertz pulse is reflected at both the air $/ \mathrm{PE}$ interface $($ depth $=0 \mathrm{~mm}$ ) and the PE/paper interface (depth $=1.8 \mathrm{~mm}$ ) owing to the changes of refractive index. For each TPI measurement a 3D data set is obtained where two axes describe vertical and horizontal spatial dimensions, and the third axis represents the depth dimension. Therefore TPI is a true 3D imaging tool and the 3D structure map of a sample can be fully reconstructed by analysing the TPI data set. Figure 1(e) shows a 3D view of the reconstructed PE pellet sample where the piece of paper buried in the pellet is revealed. The exact location of the paper inside the pellet was determined by the TPI measurement. The lateral resolution of the technique is limited by diffraction of the terahertz focus to about $150 \mu \mathrm{m}$ at $3 \mathrm{THz}$, whereas the depth resolution is mainly limited by the terahertz pulse duration to approx. $30 \mu \mathrm{m}$.

\subsection{Coating layer thickness and quality}

TPI measurements have also been analyzed to extract both coating layer thickness and interface quality of a sample. The sample is a piece of $120 \mu \mathrm{m}$ thick plastic sheet embedded in a PE pellet, as shown in the inset of Figure 2. Figure 2(a) shows the B-scan image of the sample. With the knowledge of the refractive index (pre-determined from terahertz transmission measurement), the thickness of the plastic sheet was determined to be $119 \mu \mathrm{m}$, which is very close to the $120 \mu \mathrm{m}$ as measured by a calibrator. In addition, the layer thickness of the plastic sheet is found to be fairly uniform, with a standard deviation of about $3 \mu \mathrm{m}$. However, the quality factor, defined as the amplitude ratio of the first and the second reflection peak of the terahertz waveform, varies a lot across the PE/plastic interface. This suggests that the physical contact between the plastic sheet and the PE material is not uniform. Note that the quality factor was mainly 

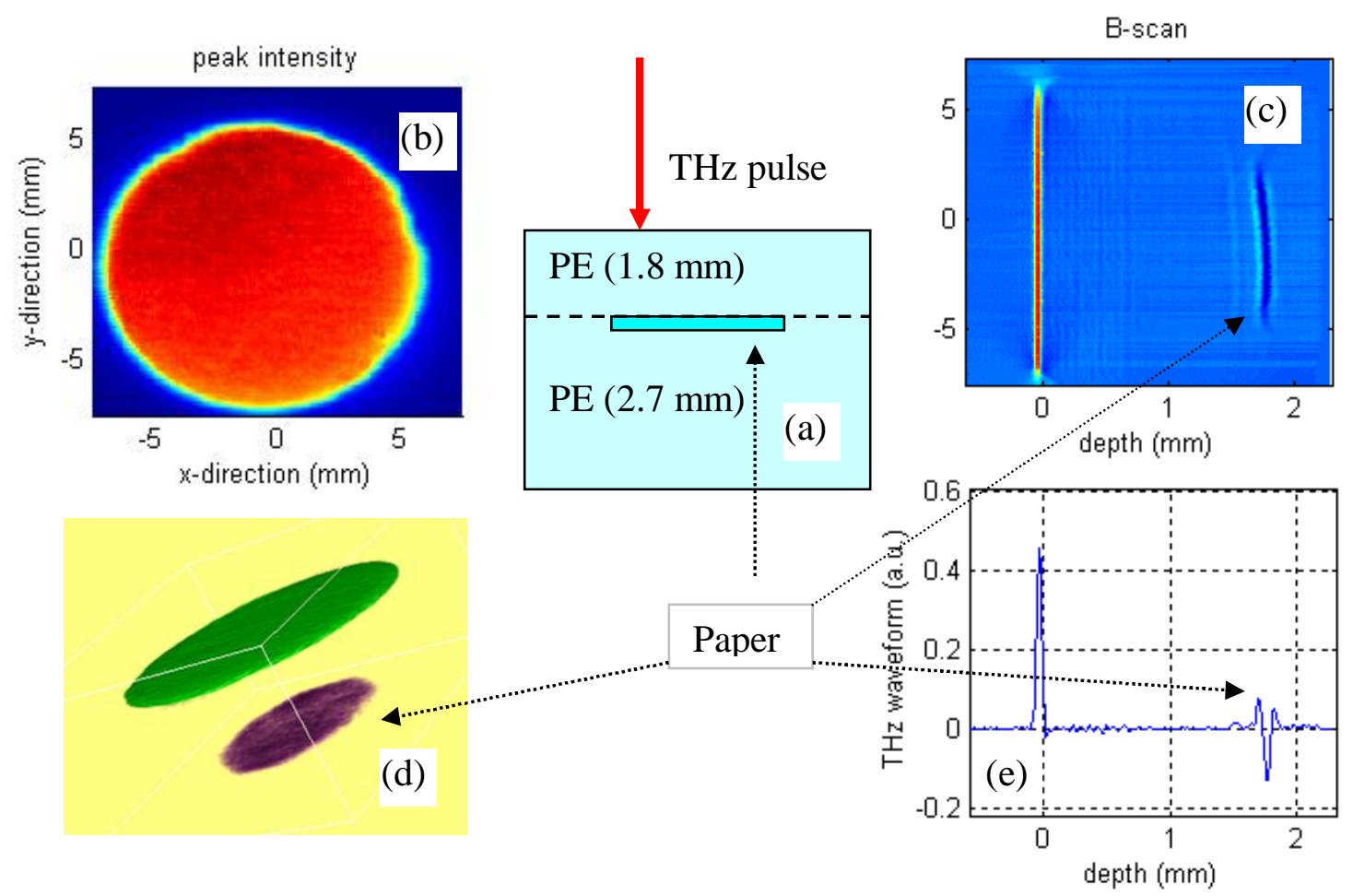

Figure 1. (a) Schematic diagram of the sample, (b) terahertz image constructed using the peak intensity of the measured THz signal, (c) B-scan image (cross section along x-direction) showing the depth information, (d) 3D view reconstructed from the TPI data, (e) example terahertz waveform showing the pulse reflections from both the surface and subsurface.
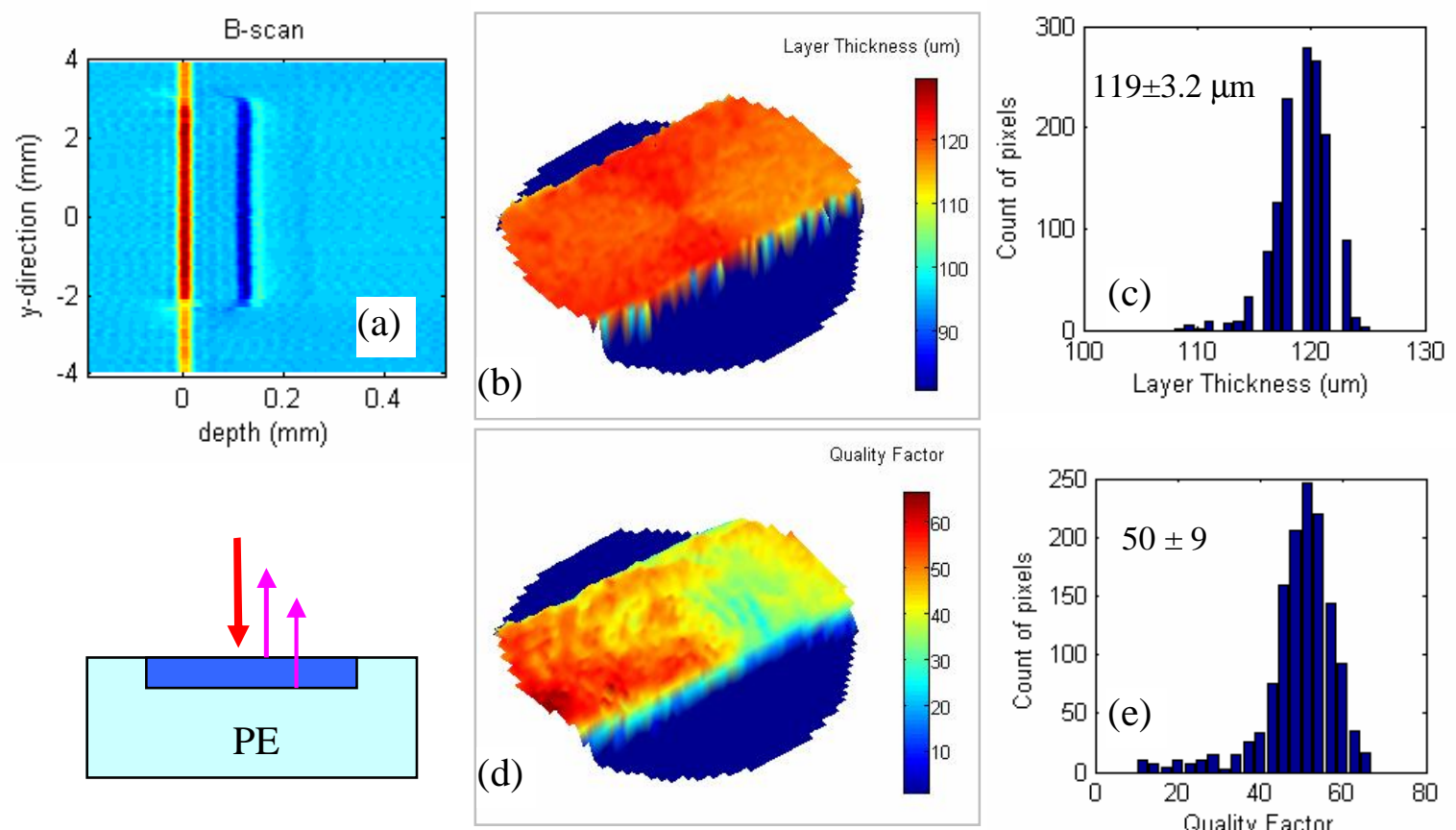

(d)

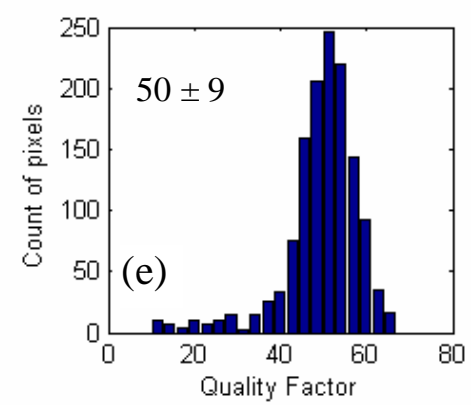

Figure 2. (a) B-scan terahertz image of the sample, (b) the distribution, (c) layer thickness histogram determined from TPI measurement, (d) distribution of the interface quality and (e) interface quality histogram extracted from the TPI measurement. Inset at bottom left shows a schematic diagram of the sample. 
determined by the change of the refractive index at the interface. Therefore, the TPI measurements provide useful information on coating layer thickness, integrity, and uniformity and the layer interface quality. Further TPI measurements on pharmaceutical tablets suggest that the quality factor and the layer thickness provide vital information on the dissolution and drug breaches at the layer interface.

\subsection{Principal Component Analysis for data compression}

As mentioned in the previous sections, the TPI measurement involves the acquisition of a 3D TPI data set. The data size of a terahertz image comprising $200 \times 200$ pixels will be 160 Mbyte because each pixel consists of a terahertz waveform with 512 points and each floating point number requires 8 bits. For some practical applications the data size could be much larger. Therefore it is of general importance to study different ways for manipulating and storing these large TPI data sets. Here we show that principal components analysis (PCA) is a powerful tool for reducing the TPI data size without losing any valuable information in the terahertz data.

PCA is a classical technique to reduce the dimensionality of a data set by transforming to a new set of variables (the principal components) that summarize the features of the data [15]. For temporal terahertz waveforms recorded for a tablet with layered structures, the main terahertz reflection peak from the tablet surface appears in the waveform of all pixels, and further terahertz reflection peaks may also appear in the waveforms of most other pixels. These are common features for a TPI data set recorded on a tablet sample. This suggests that PCA should be a very efficient tool for analysing TPI data. We developed a PCA algorithm to analyze TPI data [14] and found that the TPI data can be compressed dramatically whilst retaining most essential information on layer thickness and interface quality. As an example, Figure 3 shows the results of comparison of the layer thickness results obtained by analyzing the original TPI data and the compressed data. The TPI data size has been dramatically reduced by a factor greater than 16 from 160 Mbyte to less than 10 Mbyte without losing any essential information on coating layer thickness. In fact, the signal-tonoise ratios of the terahertz waveforms after compression are improved over the original data. This is because the noise appears randomly at different depths for different pixels and therefore is not selected by the PCA algorithm which only summarizes the common features of terahertz waveforms at all pixels.

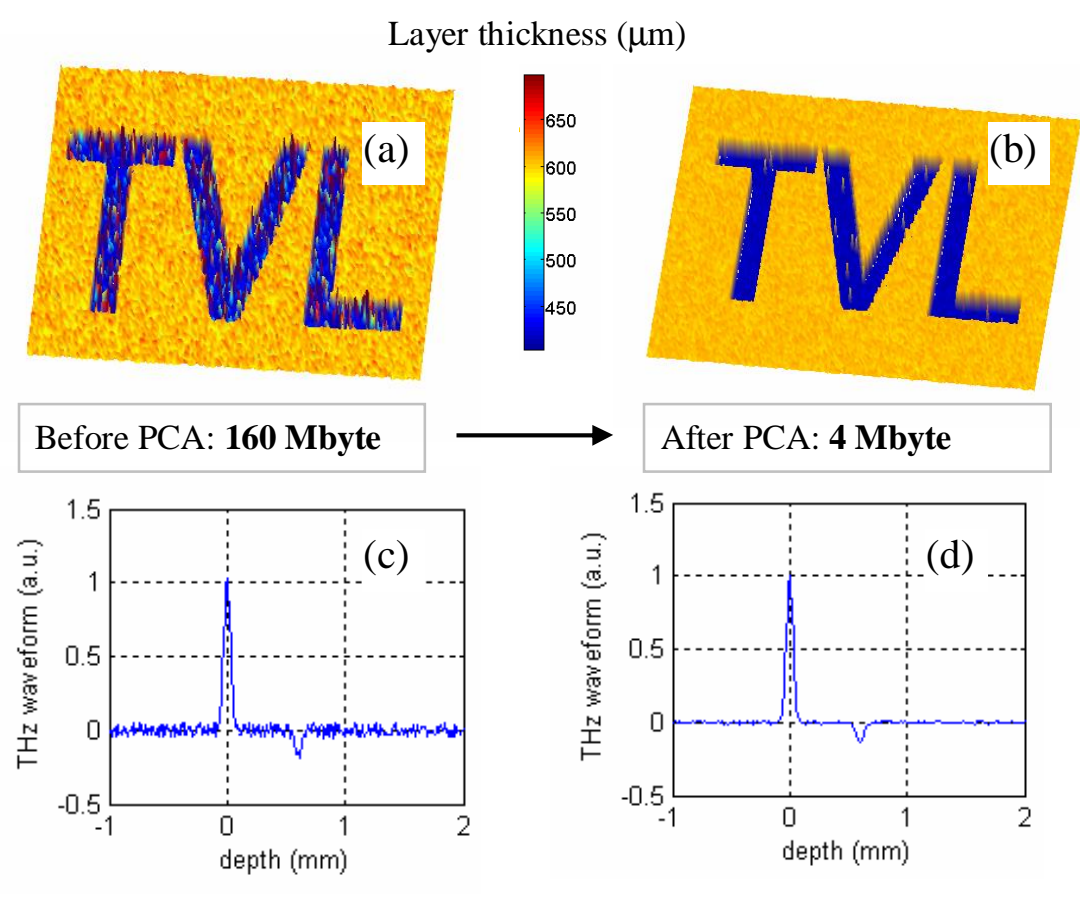

Figure 3. Results of compressing TPI data using PCA to reduce the data size from 160 Mbyte to less than 10 Mbyte whereas all useful information was retained. Distribution of layer thickness extracted from original TPI data (a) and from compressed TPI data set (b). Terahertz waveforms at a single pixel for original data (c) and after PCA compression (d). Note that the noise was automatically removed by the PCA data compression process whilst the peak positions/amplitudes are unchanged. 


\section{SPECTRAL ANALYSIS FOR 3D CHEMICAL MAPPING}

One of the most predominant features of TPI is its capability for spectroscopic discrimination. In previous sections, we have shown how time-domain TPI data may be used for tablet coating analysis. By Fourier transforming the timedomain TPI data into frequency-domain, one can obtain an additional TPI data set in the spectral frequency dimension. These spectral terahertz images contain comprehensive information on both spatial and chemical distribution of the sample. We will show in this section how these spectral TPI data can be used for detecting and identifying explosives and for mapping the 3D chemical distribution of a sample.

\subsection{Detection and identification of explosive}

Many materials of security interest such as explosives and illicit drugs have characteristic spectral features in the terahertz range [4]. Figure 4 shows the absorption spectra of lactose and 1,3,5-trinitro-1,3,5-triazacyclohexane (RDX) extracted from a TPI reflection measurement. In the spectral range $5 \mathrm{~cm}^{-1}$ to $70 \mathrm{~cm}^{-1}$ lactose has two major absorption features, centred at $18 \mathrm{~cm}^{-1}$ and $46 \mathrm{~cm}^{-1}$ whilst RDX has a major absorption feature at $26 \mathrm{~cm}^{-1}$. , These spectral fingerprints enable us to map out the distribution of individual chemicals, in this case lactose and RDX, in a sample. The sample used here comprised a pure lactose pellet and a piece of RDX, both mounted at the terahertz focus. Figure 4(a) shows the time-domain terahertz image generated from the peak amplitudes of the measured waveforms. Contrast between the lactose and RDX signals suggests that lactose and RDX have different refractive index values. However, from the time-domain terahertz image alone specific areas cannot be identified as lactose or explosive. Figures 4(b) and 4(c) show chemical mapping terahertz images obtained using component spatial pattern analysis based on the spectral fingerprints of lactose and $\operatorname{RDX}[14,16]$. The lactose pellet and piece of RDX explosive are clearly detected, located, and identified. This provides a very promising alternative way for detecting and identifying explosives and illicit drugs.
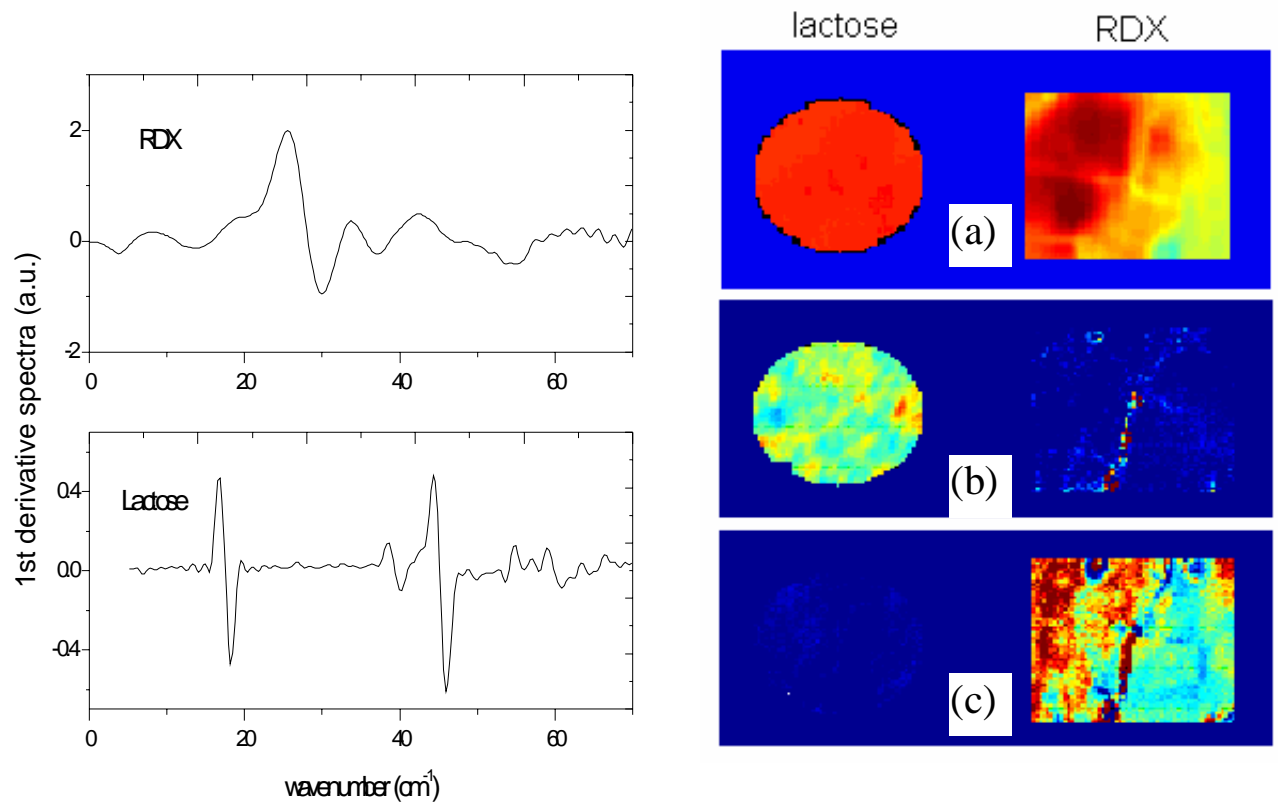

Figure 4. Left: First derivative of absorption spectra of RDX and lactose. Right: (a) terahertz time-domain image of peak amplitudes and (b) the false-colour terahertz chemical mapping image showing the spatial distribution of RDX. 


\subsection{D chemical mapping}

In the previous sections we demonstrated that the terahertz data obtained using TPI contains rich information describing the structure and composition of a sample. The change of refractive index at interfaces of a sample with layered structures produces multiple reflection peaks in the terahertz waveforms, and these permit non-destructive analysis of the coating layer (e.g. coating thickness and quality) and for detecting and locating subsurface objects. On the other hand, the characteristic spectral absorption features of different chemicals can also be extracted from TPI data, and this has been used for detecting and identifying substances including RDX explosive. In this section, we demonstrate that by analyzing both time-domain and frequency-domain TPI data a true 3D chemical map of a sample, i.e. a structural and compositional 3D view of the sample, can be obtained.

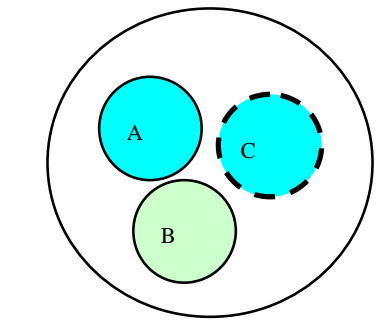

(a)

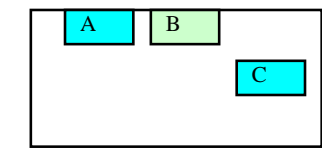

(b)

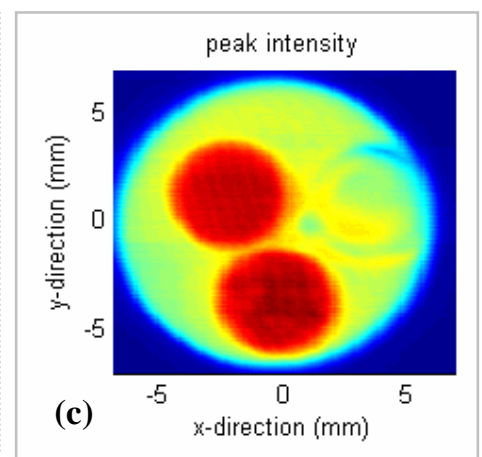

Figure 5. (a) Schematic diagram of a $13 \mathrm{~mm}$ diameter PE pellet sample formed by embedding three pre-fabricated $5 \mathrm{~mm}$ diameter pellets into compressed PE powder. . Areas A and C are pure lactose while Area B is pure tartaric acid. (b) Schematic representation of the depth profile of the sample. Note that pellet $\mathrm{C}$ is buried $1 \mathrm{~mm}$ beneath the surface of the PE pellet. (c) Time-domain terahertz image constructed using the peak amplitude of the terahertz waveform.
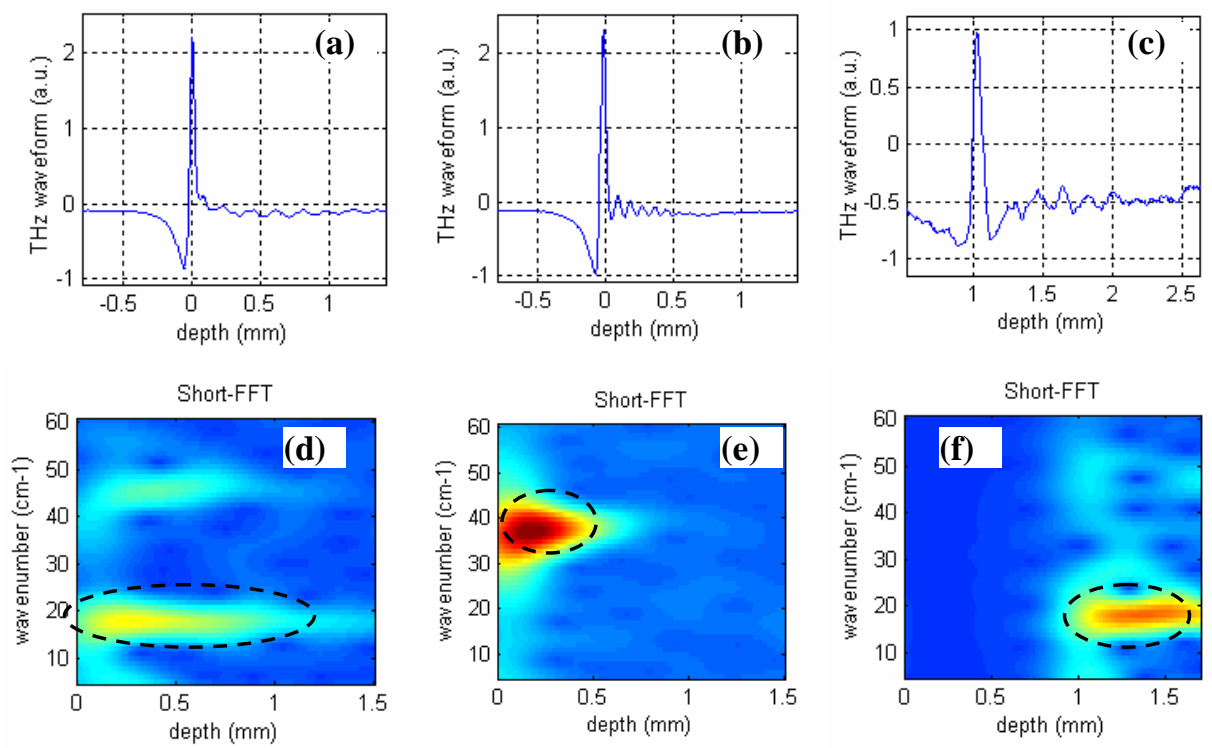

Figure 6. Typical terahertz waveforms obtained from TPI measurement of (a) area A, (b) area B, and (c) area C of the sample pellet. The results of spectral content analysis of the corresponding terahertz waveforms using timepartitioned Fourier transform are shown in (d)-(f), revealing the chemical signatures of lactose and tartaric acid. Note that the regions enclosed by dotted lines indicate the occurrence of lactose (absorption feature at $18 \mathrm{~cm}^{-1}$ ) and tartaric acid (absorption feature at $38 \mathrm{~cm}^{-1}$ ). 
The sample used here comprises three pellets, two of pure lactose and one of pure tartaric acid, imbedded in a PE pellet, as shown in Figures 5(a) and (b). The sample was prepared as follows. Pure lactose and tartaric acid powders were compressed under 2-tons using a hydraulic press (Specac, UK) to form two lactose pellets and one tartaric acid pellet, all of $5 \mathrm{~mm}$ diameter. These pre-fabricated pellets were then used together with high-density PE powder to form a larger PE pellet of diameter $13 \mathrm{~mm}$, with one lactose pellet and one tartaric acid pellet at the surface and the other lactose pellet $1 \mathrm{~mm}$ below the surface as shown in Figure 5(b). The PE powder acts as a pellet binder and is spectroscopically inert. Figure 5 (c) shows an example time-domain terahertz image which is constructed using the peak amplitudes of the terahertz waveform. Lactose and tartaric acid have higher refractive index values than PE and reflect terahertz more strongly at the air/sample interface. As a result, areas containing lactose and tartaric acid appear much brighter than PE areas.

Figures 6(a)-(c) show typical terahertz waveforms obtained for the three different areas A, B, and C corresponding to lactose, tartaric acid and buried lactose, respectively. Ripple-like oscillations appear immediately after the main terahertz pulse and these oscillations are caused by resonance absorption of the sample. Note that the terahertz pulse in Figure 6(c) is delayed by approx. $1 \mathrm{~mm}$ compared to the terahertz pulses shown in Figures 6(a) and 6(b). This timedelay provides additional depth information about the sample. For the purpose of $3 \mathrm{D}$ chemical mapping, timepartitioned Fourier transform [17] of the terahertz waveform in Figures 6 (a)-(c) were calculated, giving a spectral content map for each terahertz waveform (pixel) as shown in Figures 6 (e) and 6(f). The chemical signatures of lactose at $18 \mathrm{~cm}^{-1}$ and tartaric acid at $37 \mathrm{~cm}^{-1}$ were clearly revealed, together with their depth distribution. With the knowledge of the spectral map and the additional depth information provided in terahertz waveform, one can construct a full 3D structural and compositional chemical map of the sample, as shown in Figures 7 (a)-(e). This 3D chemical mapping not only specifies where the object is, but also tells what kind of chemical it is.

We note that ultrasonic techniques have been used for non-destructive detection of subsurface structures but these lack the specificity required for chemical mapping. On the other hand, infrared spectroscopy provides spectral features for chemical mapping but it cannot penetrate into most pharmaceutical materials nor most common packaging and clothing materials. Therefore infrared is typically limited to surface chemical mapping rather than 3D chemical mapping. The TPI technique, as demonstrated here, has a unique combination of properties in that terahertz waves propagate through many barrier materials and many chemicals of interest show characteristic spectral features in the terahertz region. These unique features of TPI make it an ideal tool for $3 \mathrm{D}$ chemical mapping.

5.

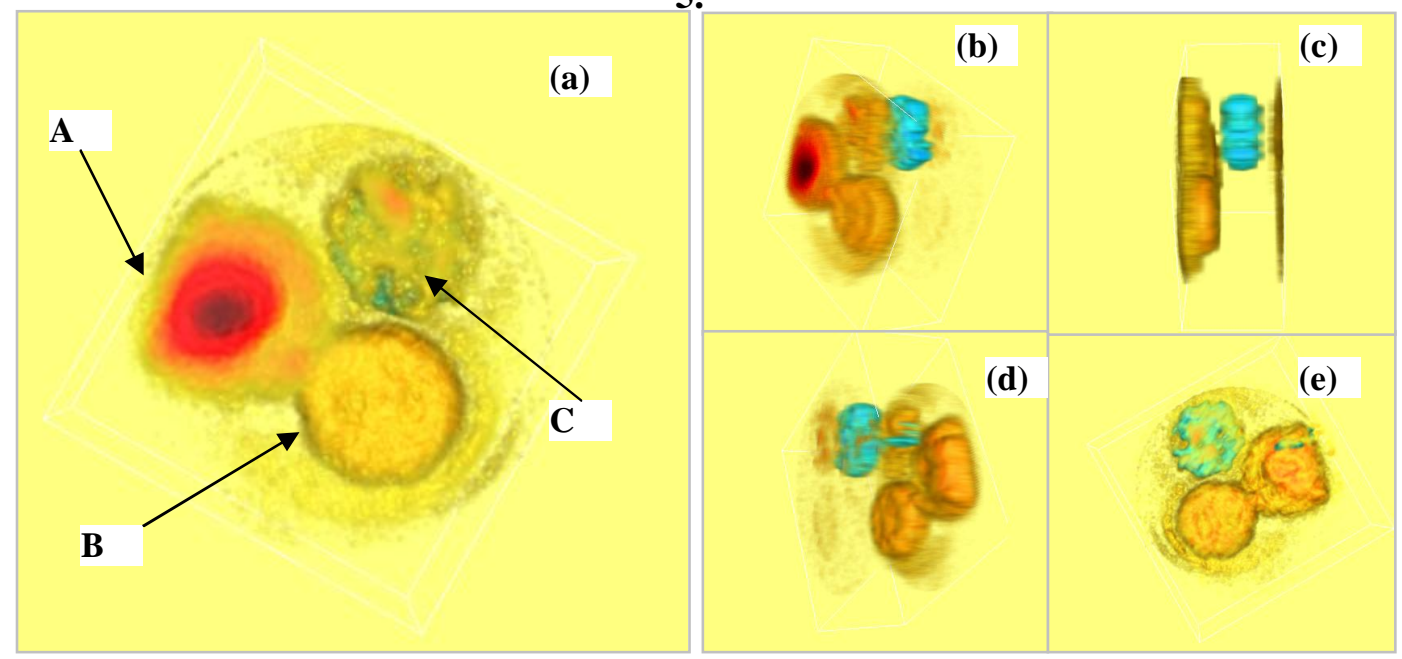

Figure 7 Three-dimensional view of the PE pellet sample reconstructed by analysing both temporal time-domain and spectral TPI data sets, (a) top view with A, B and C referring to lactose area, tartaric acid area and buried lactose area. (b) 45 degree side view, (c) 90 degree side view, (d) 135 degree side view, (e) bottom view. 


\section{Conclusions}

In this article we have demonstrated the capability of TPI for non-destructive mapping of the structural and compositional properties of samples. The applications demonstrated include: (1) inspecting subsurface structure, (2) quantifying tablet coating thickness and analyzing interface quality, (3) detecting and identifying explosives through spectral analysis, and (4) 3D chemical mapping of the spatial distribution of individual chemicals in a multi-chemical sample. The technique is unprecedented in that it is non-destructive, non-invasive, and completely safe. Furthermore, terahertz waves propagate through many pharmaceutical coating materials and barrier materials such as clothing and packaging, and many chemicals show characteristic spectral features in the terahertz range. These unique features make TPI an ideal tool for 3D chemical mapping.

\section{ACKNOWLEDGEMENTS}

Parts of this work were carried out under contract for the UK Government whose support is gratefully acknowledged. The authors thank B. E. Cole (TeraView Ltd) for helpful discussions.

\section{REFERENCES}

1. E.N. Lewis, P.J. Treado, R.C. Reeder, G.M. Story, A.E. Dowrey, C. Marcott, and I.W. Levin, Anal. Chem. 67, 337 (1995).

2. J.L. Koening, S.Q. Wang, and R. Bhargava, Anal. Chem. 73, 360A (2001).

3. F.C. Clarke, M.J. Jamieson, D.A. Clark, S.V. Hammond, R. D. Jee, and A.C. Moffat, Anal. Chem. 73, 2213 (2001).

4. M.C. Kemp, P.F. Taday, B.E. Cole, J.A. Cluff, A.J. Fitzgerald, and W.R. Tribe, SPIE 5070, 44 (2003).

5. T.D. Dorney, R.G. Baraniuk, and D.M. Mittleman, J. Opt. Soc. Am. A 18, 1562 (2001).

6. B. Ferguson and X.-C. Zhang, Nat. Mater. 1, 26 (2002).

7. M.C. Beard, G.M. Turner, and C. A. Schmuttenmaer, J. Phys. Chem. B 106, 7146 (2002).

8. M. Walther, B. Fischer, M. Schall, H. Helm, and P. Uhd Jepsen, Chem. Phys. Lett. 332, 389 (2000).

9. Y.C. Shen, P.C. Upadhya, A.G. Davies, and E.H. Linfield, Appl. Phys. Lett. 82, 2350 (2003).

10. P.F. Taday, I.V. Bradley, D.D. Arnone, and M. Pepper, J. Pharmaceutical Sci. 92, 831-838 (2003).

11. C.J. Strachan, T. Rades, D.A. Newnham, K.C. Gordon, M. Pepper, and P.F. Taday, Chem. Phys. Lett. 390, 20 (2004).

12. A.J. Fitzgerald, B.E.Cole, and P.F. Taday, J. Pharmaceutical Sci. 94, 177-183 (2005).

13. V.P Wallace, A.J Fitzgerald, S Shankar, N Flanagan, R.J Pye, J Cluff, and D.D. Arnone, Brit. J. Dermatol. 151, 424 (2004).

14. Y.C. Shen, P.F. Taday, and M.C. Kemp, SPIE 5619, $82-89$ (2004).

15. I.T. Jolliffe, Principal Component Analysis (Springer, New York, 1986).

16. Y. Watanabe, K. Kawase, T. Ikari, H. Ito, Y. Ishikawa, and H. Minamide, Appl. Phys. Lett. 83, 800 (2003).

17. Y.C. Shen, P.C. Upadhya, A.G. Davies, and E.H. Linfield, Phys. Rev. B 69, 235325 (2004). 